\title{
Correspondence
}

\section{miRDeathDB: a database bridging microRNAs and the programmed cell death}

\author{
Cell Death and Differentiation (2012) 19, 1571; doi:10.1038/cdd.2012.87; published online 29 June 2012
}

Dear Editor,

MicroRNAs (miRNAs) are a class of small non-coding RNAs, which negatively regulate protein-coding genes and have been associated to almost all known physiological and pathological processes. ${ }^{1}$ Apoptosis, autophagy and programmed necrosis are three distinct yet intimately connected functional modules of programmed cell death (PCD). In the past few years, increasing evidences suggested that aberrant expression of miRNAs regulate different subroutines of PCD. ${ }^{2,3}$ Exploring the roles of miRNAs in PCD network has attracted interests of researchers from a wide range of areas. Therefore, we developed miRDeathDB (http://rna-world.org/ mirdeathdb/), a knowledge depository that integrates information for experimentally identified miRNAs and their targets in PCD network.

In total, 210 records covering 86 miRNAs and 95 proteincoding genes were collected in five model species. Users can search the PCD-associated miRNAs and their targets based on gene principal symbols. In addition, users can retrieve plenty of genomic, proteomic and disease-associated data via links to external resources. Detailed information on database construction and usage can be found in Supplementary File.

miRDeathDB provides a valuable resource for archiving PCD knowledge and can be used to understand general features of miRNAs in cell death network. For example, we found that miR-21 regulated eight cell death-related protein, while BCL2 was regulated by 16 miRNAs (Supplementary Figure 1). Similarly, autophagic protein BECN1 and MAP1LC3B were also targeted by multiple miRNAs (Supplementary Figure 1). These examples indicated a single miRNA could target multiple cell death-related proteins and one cell death-related protein could be regulated by multiple miRNAs, highlighting the plasticity and complexity of the regulatory relationships between miRNAs and cell death-related proteins.

Cross-talk among different modules of PCD is intricate and fine tuned. Accumulating evidences suggested cell death subroutines share several protein-coding regulators that are essential for their respective executions. ${ }^{2}$ It seems also true for miRNA regulators. As shown in Supplementary Figure 1, miR-101, miR-204 and miR-221 have dual roles in apoptosis and autophagy. On the basis of these observations, we recently proposed that miRNAs may work as molecular switches between apoptosis and autophagy. ${ }^{3}$ miRNAs can target common protein-coding regulators of these two cell death modules, or simultaneously modulating multiple targets, which function either in autophagy or apoptosis. Although the roles of miRNAs in programmed necrosis still remain elusive at present, recent studies have elucidated several shared proteincoding components of the machineries regulating necrosis, autophagy and apoptosis. ${ }^{4}$ Therefore, it would be interesting to examine whether or not miRNAs stand at the cross-road of necrosis and other subroutines in PCD network.

Several cell death-related databases centered on proteincoding genes have been built recently. ${ }^{5,6}$ Complemented with these useful databases, we constructed miRDeathDB and established a novel link between miRNAs and PCD. To keep up with these two rapidly growing research fields, we will continuously curate the literature data and update miRDeathDB. We hope this resource will become a bridge between the miRNAs and cell death research and stimulate further interest on the role of miRNAs in PCD network.

\section{Conflict of Interest}

The authors declare no conflict of interest.

Acknowledgements. This work is supported by the science foundation of education department of Henan province (No. 2011A180009) and a grant from Henan University of Technology (\#2009BS040).

$J X u^{*, 1,4}$ and $Y-H L i^{2,3,4}$

${ }^{1}$ College of Bioengineering, Henan University of Technology, Zhengzhou 450001, China;

2 Institute of Cardiovascular Sciences and Key Laboratory of Molecular Cardiovascular Sciences, Ministry of Education, Peking University Health Science Center, 38 Xueyuan Road, Haidian District, Beijing 100191, China and

${ }^{3}$ Scientific Data Center, Computer Network Information Center, Chinese Academy of Sciences, Beijing 100190, China

* Corresponding author: Jianzhen Xu, College of Bioengineering, Henan University of Technology, Zhengzhou 450001, China. Tel: +86 371 67756928; Fax: +80 371 67756513; E-mail: xujz0451@gmail.com

${ }^{4}$ These authors contributed equally to this work.

1. Bartel DP. Cell 2009; 136: 215-233.

2. Eisenberg-Lerner A et al. Cell Death Differ 2009; 16: 966-975.

3. Xu J et al. Autophagy 2012; 8: 1-10.

4. Walsh CM, Edinger AL. Immunol Rev 2010; 236: 95-109.

5. Diez J et al. Cell Death Differ 2010; 17: 735-736.

6. Homma K, Suzuki K, Sugawara H. Nucleic Acids Res 2010; 39: D986-D990. 